\title{
Horizons/Théâtre
}

Revue d'études théâtrales

10-11 | 2017

Genre et arts vivants

\section{Questions pour une méthodologie féministe dans l'histoire du théâtre}

\section{Tracy Davis}

\section{(2) OpenEdition}

1 Journals

\section{Édition électronique}

URL : http://journals.openedition.org/ht/556

DOI : $10.4000 /$ ht.556

ISSN : 2678-5420

\section{Éditeur}

Presses universitaires de Bordeaux

\section{Édition imprimée}

Date de publication : 1 juillet 2017

Pagination : 175-201

ISSN : 2261-4591

\section{Référence électronique}

Tracy Davis, «Questions pour une méthodologie féministe dans l'histoire du théâtre », Horizons/ Théâtre [En ligne], 10-11 | 2017, mis en ligne le 01 juillet 2018, consulté le 20 juillet 2019. URL : http:// journals.openedition.org/ht/556 ; DOI : 10.4000/ht.556

\section{(c)}

La revue Horizons/Théâtre est mise à disposition selon les termes de la Licence Creative Commons Attribution - Pas d'Utilisation Commerciale - Pas de Modification 4.0 International. 


\section{Questions pour une méthodologie féministe dans l'histoire du théâtre}

Dans presque tous les domaines des sciences humaines et sociales, la recherche féministe est devenue un élément majeur du débat scientifique. Les remarques qui suivront sur une méthodologie féministe pour l'histoire du théâtre intègrent quelques-unes des questions qui ont été posées par les sociologues, les critiques littéraires, les historien.ne.s des femmes, et les sémioticien.ne.s mais ne cherchent à imiter aucun de ces groupes en particulier. Bien que notre approche soit fondamentalement interdisciplinaire, elle échappe au pur éclectisme du fait de sa fidélité à une idéologie politique cohérente, à savoir le féminisme. Nos propositions s'appuient en partie sur des exemples de travaux historiques féministes sur le théâtre (la série d'articles et de livres repérés autour de 1972), en partie sur des écrits méthodologiques sur l'histoire des femmes en tant que défis intellectuels (excellemment résumés par Lerner $)^{1}$, et en partie sur une préférence personnelle pour la façon dont le champ devrait prendre forme.

De nombreuses recherches actuelles portent sur les groupes de théâtre féministes et adoptent une méthodologie féministe dans leur création et mises en scène - essentiellement une entreprise descriptive et non analytique, qui ne remodèle pas le travail de l'historien.ne. Dans une communauté universitaire curieuse, mais sceptique à l'égard du féminisme, une certaine pression pèse sur nos conclusions ; il serait attendu qu'elles soient miraculeusement justes dès la première tentative. Cela explique que nous utilisons habituellement des modèles de recherche conventionnels saupoudrés de théorie littéraire et de philosophie féministes. Étant donné que le théâtre a été utilisé comme un outil féministe d'agitation-propagande et d'éducation pendant la majeure partie de ce siècle (d'abord dans les campagnes des suffragettes et de façon continue depuis le milieu des années 1960), que les débats féministes se répandent dans le milieu universitaire et qu' un grand nombre de praticien.ne.s et d'historien·ne.s du théâtre s'intéressent au sujet, il ne semble pas prématuré d'entamer une discussion d'ordre méthodologique. 


\section{Identifier le sujet}

La crainte est vive, dans les études théâtrales, de « ségréguer » les femmes, y compris chez les rédacteurs en chef de revues qui leur consacrent des numéros spéciaux (d'où l'introduction apologétique et sans substance de The Drama Review, T86) ${ }^{2}$. Les critiques féministes qui travaillent à partir de modèles gynocritiques élaborés sont plus avancé.e.s dans leur application des théories féministes, mais ni l'art dramatique, ni la mise en scène de textes ne fait habituellement partie de leur objet d'étude ${ }^{3}$. Par conséquent, les avancées critiques découlent, aujourd'hui encore, des sciences du langage plutôt que des études théâtrales. Toutefois, le travail récent de Janelle Reinelt sur la théorie brechtienne et l'écriture théâtrale britannique, et des articles comme ceux de Phyllis Rackin qui examinent les diverses analyses de l'androgyne (des garçons qui incarnent des héroïnes) sur la scène de la Renaissance, suggèrent que cette lacune est désormais en partie comblée ${ }^{4}$.

Comme dans les études cinématographiques, l'histoire de l'art et l'anthropologie de la danse, la représentation du corps de la femme et la socialisation des femmes sont des sujets d'une importance essentielle pour tou.te.s les féministes. Les interprétations des sens et des significations peuvent varier, mais il semble y avoir un consensus autour du fait que les représentations de la sexualité et du genre sont et ont été des constructions masculines (représentations de, mais non par les femmes) et que l'érotisme est un intérêt primordial pour l'histoire féministe des spectacles. L'historien de la danse Frank Aschengreen, par exemple, soutient qu'après deux cents ans de domination par les danseurs hommes, le ballet romantique français a pratiquement supprimé le masculin, le remplaçant par une nouvelle esthétique où le démonisme se serait conjugué à l'érotisme chez des ballerines qui éveillaient directement les sens ${ }^{5}$. Ann Daly propose une analyse analogue des chorégraphies de George Balanchine, qui glorifient une image passive, manipulée de la féminité . Jill Dolan décrit la façon dont les expériences que mènent les femmes dans la performance, et le cabaret lesbien révèlent la rigidité de nos conceptions de la représentation, les performances érotiques conçues par les femmes pouvant briser ce moule. Ailleurs, j'ai abordé la question du langage partagé par la pornographie et la scène victoriennes, soutenant que l'intérêt de la pornographie pour les actrices (fictionnelle et réelle) influençait et reflétait à la fois la façon dont les hommes percevaient le spectacle ${ }^{8}$. Ainsi, les travaux qui peuvent être considérés comme un modèle pour la méthodologie de l'histoire du théâtre féministe, quoique limités en nombre, sont en expansion. 
Nancy Reinhardt et Claudia Johnson ont montré l'importance de la classe sociale et du genre dans la littérature dramatique, et dans les bâtiments des théâtres et les salles de spectacle ${ }^{9}$, mais en général les dénommées histoires culturelles des artistes et des genres populaires (habituellement illégitimes) forment un des champs les plus méthodologiquement exigeants des études théâtrales. Les questions de classes sociales imprègnent les travaux de Peter Bailey [auteur d'une histoire sociale du music-hall], de Penny Summerfield [historienne des femmes], et de Eileen et Stephen Yeo [historien.ne.s de la culture populaire et des conflits de classes] : les distinctions de classe dans les amphithéâtres, dans les garde-robes, et dans les chansons des music-halls de l'époque victorienne sont interprétées comme des éléments d'analyse convaincants voire incontestables. La plupart des féministes conviendraient que les distinctions de classe interagissent avec la représentation et la réception des spectacles, et que leur prise en compte est essentielle dans l'écriture de l'histoire. Sheila Rowbotham avance, cependant, que « nous ne sommes pas d'une part, des êtres sexué.e.s dans la famille, et d'autre part, des membres d'une classe sociale, dans la société civile, pour l'État, et au travail $\gg:$ le capitalisme et le patriarcat s'entremêlent, affectant les femmes dans « leur perception d'elles-mêmes et des autres, leur travail, leurs habitudes et leur sexualité, leur participation à l'organisation, leurs réponses face à l'autorité, à la religion, à l'État, et l'expression de leur créativité dans l'art et la culture ${ }^{10} \gg$.

Dans un essai sur Jenny Hill, une chanteuse et danseuse de l'époque victorienne tardive qui s'est produite dans des rôles aussi bien comiques que sérieux, J. S. Bratton montre la façon dont ces éléments d'analyse peuvent être introduits dans l'histoire du music-hall. Elle réexamine les mythes contemporains et posthumes de Hill à la lumière des considérations économiques, matérielles, sociales et professionnelles de son statut de femme : une défenseuse des intérêts des classes ouvrières (surtout des femmes), une oratrice, porte-parole acharnée dans une profession où les femmes ne faisaient que rarement entendre leur voix, une artiste reconnue dont les tentatives pour gérer sa propre carrière et investir ses revenus dans la promotion de sa carrière ont vacillé en partie à cause des limitations imposées de l'extérieur aux personnes de son sexe. D'après Bratton, ceux et celles qui ont mythifié Hill la présentent comme une jeune fille exploitée dont l'excellence dans la pratique de son art a finalement conduit à des alliances avec des hommes ambitieux, puis à un succès professionnel rapide. La révision de Bratton est importante car elle présente Hill comme une artiste à succès dont la chance supposée n'est que le résultat d'un travail acharné et de son talent, et dont 
la carrière a été continuellement façonnée par des facteurs qui échappent à son contrôle, facteurs délibérément rendus invisibles par le show-business, l'époque, et Hill elle-même. Au regard du public, Jenny Hill était « l'étincelle vitale $\gg$ (le glamour vital, vedette, énergie et expertise du divertissement populaire), mais elle était en même temps à la fois limitée et entretenue par le contrôle patriarcal des cabarets de la fin du XIX ${ }^{e}$ siècle, qui l'ont empêchée, comme de nombreuses autres femmes, de parvenir à se faire une place et à la maintenir dans le panthéon masculin des interprètes et impresarios.

Elle était bien l'employée perpétuelle, certes honorée, qui recevait des diamants d'admirateurs et collègues. Bratton analyse judicieusement l'actrice couverte de bijoux comme le signe d'être possédée plutôt que de posséder : «Elle porte l'insigne de ses honneurs déshonorants qui impressionnent les femmes moins bien loties, tandis qu'elle met en garde les hommes, dont la richesse ne correspond pas à celle de ses protecteurs du moment, de ne pas l'approcher ${ }^{11}$. » Même si, comme dans le cas de Hill, la plupart des bijoux sont offerts par ses managers et son public, et non par ses amants, « le signe reste puissant ; le patriarcat perçoit la richesse [de Hill] comme provenant de cadeaux faits par des hommes plutôt que comme la marque de la capacité des femmes à gagner leur vie ${ }^{12} \gg$. En aucun cas, le cadeau indemnise les femmes interprètes des inconvénients liés de facto à leur condition professionnelle, à savoir le contrôle qu'ont les hommes de la gestion des artistes, de l'embauche, des salaires, du répertoire, et de la réputation sexuelle des femmes.

Rina Fraticelli, Kay Carney, et Julianne Boyd (toutes comédiennes professionnelles) dressent des conclusions similaires au sujet du patronage et des infrastructures socio-sexuelles de la profession dans leurs travaux sur la sous-représentation des femmes administratrices, directrices, scénographes, dramaturges aux postes les plus prisés, de nos jours au Canada et en Amérique $^{13}$. Elles soutiennent l'idée que les trajectoires professionnelles des carrières de femmes doivent être étudiées de pair avec les orientations culturelles et professionnelles qui conduisent les hommes et les femmes vers différentes professions. Judith Lynne Hanna pousse la notion de ghetto professionnel plus loin en affirmant que les choix de carrière sont liés aux prestiges des catégories professionnelles qui sont déterminées par le genre, les femmes et les hommes homosexuels étant relégués à des emplois symboliquement et économiquement précaires, dans des champs comme la danse. En effet, le sexe, les orientations sexuelles, les constructions culturelles 
du « corps, de l'émotion, du genre » ont une incidence significative sur les profils des emplois ${ }^{14}$.

Les féministes ne s'intéressent pas seulement aux femmes en tant que catégorie biologique ; elles se réunissent autour de la conviction que le genre est une construction sociale, et que cela constitue l'élément principal de leurs analyses. Certain.e.s historien.ne.s des femmes au théâtre, comme Rosamond Gilder, Albert Auster, Claudia Johnson et Katharina Wilson, établissent une distinction sexuelle (biologique) sans nécessairement établir de distinction (sociale) de genre. Par conséquent, une perspective politique renseignée qui examinerait l'existence des personnes dont il est question dans leur ouvrage comme des êtres sociaux appartenant à un temps et à un espace spécifique fait défaut ${ }^{15}$. Des anthropologues marxistes telles que Sherry Ortner et Harriet Whitehead, qui considèrent la culture comme une idéologie qui justifie le statu quo, en mystifiant et en déformant systématiquement les sources d'oppression et d'exploitation, fournissent des pistes plus fructueuses ${ }^{16}$. Dans la tradition des Annales, Susan Dwyer Amussen documente ce processus culturel concernant l'époque moderne en Angleterre en montrant les mises en application informelles des attentes sociales vis-à-vis du genre à travers les ragots et la manipulation des réputations, qui affectent la cour, le mariage et les ruptures conjugales ${ }^{17}$. L'importance de ces facteurs dans l'histoire du théâtre - particulièrement les biographies des actrices - est généralement reconnue, mais sous-estimée par les historien.ne.s du théâtre.

\section{Approches}

La plupart des travaux publiés sur les femmes et le théatre s'inscrivent aisément dans l'une des deux catégories suivantes :

1. Des projets qui s'emploient à retrouver des données historiques sur les femmes et à combler ainsi les « blancs » de l'histoire au sujet des femmes. Cela nous mène à discuter de l'asymétrie genrée de la société et de la culture, et à envisager une histoire révisionniste, notamment sous la forme de biographies, individuelles et collectives (jusqu'à présent en général, réservées aux plus glorieuses, célèbres, et donc, aux élites atypiques de l'art). Le recouvrement et la révision prennent aussi la forme d'histoires d'actions ouvertement féministes, tout particulièrement celles de l'engagement d'actrices dans le mouvement des suffragettes et des groupes de théâtre féministes, qui mettent en lumière des activités de femmes qui contrastent avec ou s'opposent aux traditions dominantes ${ }^{18}$. 
2. Une critique littéraire féministe qui complète le travail des historien·ne.s en récupérant les pièces de théâtre « perdues » et les dramaturges non canonisées, fournissant une lecture alternative des textes, et rééditant ou publiant des pièces contemporaines de femmes. ${ }^{19}$

\section{L'histoire révisionniste}

Le recouvrement [action de retrouver ce qui était perdu] est probablement un premier pas indispensable aux recherches féministes, mais, comme le signale Lynda Nead au sujet de l'histoire de l'art, l'effort ne doit pas s'arrêter à redécouvrir des noms et à les intégrer à la discipline ; il doit se poursuivre pour remettre en question les termes, la périodisation et les catégories de la tradition universitaire ${ }^{20}$. Jusqu'à présent, le canon des travaux universitaires et la panoplie de célébrités héritées de l'histoire traditionnelle n'ont pas encore été substantiellement révisés, ainsi un travail comme celui de Nancy Cotton sur les autrices de théâtre anglaises, du quinzième au dix-huitième siècle, exceptionnel dans son attention portée aux personnages historiques mineurs et oubliés, est admirable, mais n'est pas conclusif ${ }^{21}$. Si les historien.ne.s féministes réécrivent l'histoire, la révision doit entailler [la discipline] profondément. Les « blancs » dans l'histoire ne concernent pas seulement la vie des femmes de théâtre. L'importance du théâtre comme un média culturel, le contexte social des spectacles, des interprètes, et les hypothèses inhérentes aux versions « non révisées 》 de l'histoire sont des enjeux majeurs dans l'étude des personnages publics (des femmes notamment) dont les moyens d'existence dépendaient de l'approbation de personnes privées (des hommes notamment).

Une attention plus grande apportée à des périodes de l'histoire définies en fonction de la vie et des pratiques culturelles des femmes permettrait sans aucun doute d'encourager la recherche sur le théâtre aux époques contemporaines et plus lointaines, et aux chercheurs et chercheuses, d'explorer des indices peu habituels. L'itinérance des artistes de la scène, leurs vies excentriques ne sont pas propices à la collecte, et donc au dépôt de correspondances manuscrites, journaux, et dossiers professionnels (tels que des contrats et curriculum vitae) ; cela entraîne un témoignage élitiste de l'histoire du théâtre. Les historien.ne.s du music-hall sont aidé.e.s par la quantité de partitions publiées au XIX ${ }^{\mathrm{e}}$ siècle, mais une fois encore, il y a de sérieuses difficultés d'interprétation et les documents sont conservés de manière hasardeuse. Comme l'avance Thomas Postlewait dans son article [«Autobiography and theatre history $]^{22}$, l'interprétation d'autobiographies publiées est elle aussi problématique, en partie du fait de la réticence des 
femmes à évoquer les machinations invisibles du milieu professionnel (tel que l'exclusion patriarcale et la contrainte sexuelle) et à mentionner des affaires personnelles délicates liées à des liaisons amoureuses et aux étapes de la vie d'une femme. Parfois, les sources de l'histoire féministe, imaginativement interprétées, rempliront les blancs de l'histoire. Parfois, elles y ajouteront de nouveaux chapitres et ouvrages entiers.

Peu d'éléments sont apparus pour le moment qui permettraient de réévaluer les expériences théâtrales des femmes, à l'exception d'interprètes vedettes, de femmes, productrices hors paires, ou de critiques privilégiées, mais le révisionnisme féministe n'est pas limité aux femmes dans ces rôles. Marie-Claire Pasquier ose poser la question « Pour qui se fait le théâtre ? », puis « Qui le conçoit et selon quelles modalités ? »- deux questions aussi provocantes que dérangeantes à propos de l'amour des femmes pour le théâtre et de leur participation périphérique aussi bien en tant que spectatrices que professionnelles ${ }^{23}$. Helen Chinoy et Linda Jenkins ont consciencieusement évité cette approche de chroniques-des-stars dans leur anthologie américaine et suggèrent plusieurs nouvelles directions intéressantes :

Nous avons besoin de recherches qui examinent l'image/icône visuelle sur scène telle qu'elle est conçue par le metteur en scène ou la metteuse en scène/l'interprète/le ou la scénographe. Quelles valeurs genrées sont renforcées ou créées par la seule « image scénique »? Nous avons besoin d'études plus nombreuses sur la réception du public, sur son expérience de l'événement théâtral. [... ] Y a-t-il une distinction de genre quant à la censure d'images scéniques qui remettent en question les images socialement institutionnalisées ${ }^{24}$ ?

Certains de ces défis ont commencé à être relevés. L’adéquation des femmes aux structures théâtrales et aux procédés utilisés dans les réalisations scéniques a été remise en cause par Roberta Sklar, tout comme le genre comme catégorie d'analyse dans la création et la réception a été mobilisé par Linda Jenkins et Susan Ogden-Malouf. Elles le révèlent dans la formation et l'entraînement des acteurs et des actrices, ainsi que dans les agencements « masculinistes » du langage de la mise en scène ${ }^{25}$.

$\mathrm{Ni}$ le féminisme comme théorie politique ni le féminisme comme pratique méthodologique de l'histoire ne sont tout à fait constitués en une vision unitaire : tous deux sont émergents et ont de multiples facettes; en conséquence, d'indénombrables et irréconciliables divergences théoriques sont perceptibles. Ce qui est continuellement démontré, néanmoins, est le fait que l'histoire féministe du théâtre est incompatible avec la méthodologie positiviste. Le positivisme pourrait donner lieu à une histoire viable des 
femmes, mais il reste inconciliable avec les questions de fond soulevées par les études féministes ; les faits historiques observés ne reflètent pas nécessairement le contexte politique et culturel des activités artistiques, et ils n'éveillent que trop rarement un quelconque intérêt pour leur causalité. Une histoire du théâtre qui réunit des sources primaires (textuelles, visuelles et tactiles) pour décrire, et non pour analyser la représentation conduit à ce que Vesna Pistotnik appelle « l'archéologie des formes théâtrales et l'étude de la façon dont elles ont été utilisées pour servir les besoins du texte dramatique sur scène ${ }^{26} \gg$. Les historien.ne.s féministes du théâtre se satisfont rarement des approches « récréatives » parce que le sens des réalisations scéniques nous semble primordial. Les relations réciproques entre la représentation et les facteurs psychologiques, sociologiques, biologiques et économiques [d'une époque donnée] contribuent au défi que constitue l'historiographie récente. Les féministes ajoutent à cette liste l'importance de la dimension personnelle de l'activité sociale, et insistent sur le sens politique de tout cela. Le type de questions posées dans le recueil d'histoires orales de Kathleen Betsko et Rachel Koening, par exemple, confirme l'importance du cycle de vie des femmes dans leur vie d'artiste, et suscite des commentaires de la part des femmes dramaturges sur la façon dont leur expérience personnelle est liée à une perception du monde, qui se retrouve dans leurs pièces et mises en scène ${ }^{27}$.

Conformément aux objectifs initiaux des ateliers d'histoire du Ruskin College $^{28}$ [fondées en 1966, les étudiant.e.s y étaient encouragé.e.s à effectuer des recherches historiques fondées sur leurs connaissances d'un terrain ou d'une population, et à les présenter aux côtés d'universitaires reconnu.e.s lors d'ateliers annuels], les historien.ne.s féministes du théâtre semblent déterminées à accorder du crédit aux modes de résistance informels et aux révolutions de petite envergure, à s'appuyer sur l'observation participante et l'histoire orale (témoignages et entretiens), et souvent, à écrire, des histoires situées locales ou régionales. L'idée fondamentale est de donner la parole à ce que les individus, les cultures et l'histoire elle-même passeraient autrement sous silence. Parce que le silence n'est pas l'absence, cette démarche n'est pas seulement crédible, mais véritablement un sujet légitime de recherche historique. En historicisant le silence (et ce qui crée le silence ${ }^{29}$ ) de minorités raciales, de cultures ethniques, et de classes [...], nous explicitons ce qui est connu des femmes, mais qui n'est pas évoqué ou évocable dans la tradition dominante. Par exemple, en interviewant des femmes dramaturges, Koenig découvrit que « le censeur, agissant sur soi et dans la société était à la fois désireux et contraint de passer sous silence les éléments de travail qui sont 
liés aux expériences et au vécu des femmes, ces éléments qui critiquaient ou remettaient en question le pouvoir et l'autorité masculine, ou encore ces éléments qui révélaient véritablement la vulnérabilité masculine ${ }^{30} \gg$. La tâche des historien.ne.s féministes est de s'attaquer à l'impulsion de la censure, de valider l'expérience vécue, et de mettre en lien les femmes et le travail, et le travail avec le monde en général.

\section{La critique féministe littéraire}

La distinction entre l'art dramatique et les pièces de théâtre écrites est fondamentale : de la même manière que le personnel est politique (intégratif et réactif), le théâtral est rhétorique (persuasif et incarné). Tandis que les poétiques féministes du texte dramatique pourraient se révéler, avec le temps, essentielles à la méthodologie de l'histoire du théâtre, prendre en compte les conditions de création de la mise en scène et les nombreuses forces qui façonnent les décisions artistiques et la réception sociétale du théâtre doit être associée à un courant de la critique contemporaine davantage axé sur l'herméneutique et l'exégèse ${ }^{31}$. L'histoire du théâtre féministe intègre nécessairement l'histoire littéraire en interrogeant la façon dont l'art (ou le non-art) est lié aux goûts de son public et aux genres prédominants de son espace-temps, comme le fait Lisa Jardine dans son extraordinaire étude des femmes et de la littérature dramatique dans l'Angleterre de la Renaissance ${ }^{32}$.

Le conservatisme du courant dominant des études théâtrales provient d'une préoccupation tenace (après 2300 ans) pour la terminologie aristotélicienne de La Poétique : la classification des genres poétiques et théâtraux faite par Aristote, et sa division de la structure des évènements dramatiques, n'a pas grand-chose à voir avec l'expérience théâtrale de la femme « ordinaire » (ou même de la femme exceptionnelle), que ce soit en tant que spectatrice, actrice ou dramaturge. En mettant à jour les concepts genrés de forme, de contenu, et d'excellence dans la littérature dramatique, Reinhardt et Carol Gelderman ont soulevé certaines questions utiles aux critiques littéraires (dont la structuration linéaire, le débat viril de l'agon [scène de combat et de débat dans la tragédie grecque], et l'individualisme masculin). Dinah Leavitt, Karen Malpede, et les numéros spéciaux de The Drama Review et de la Canadian Theatre Review, en rendant hommage à ce que les femmes ont choisi de faire en dehors des pratiques [théâtrales] habituelles, ont donné à ces publications une valeur historique ${ }^{33}$. Les recherches du Projet Magdalena autour des processus créatifs et du langage expressif des femmes réalisent, dans le cadre d'un «laboratoire », un projet analogue ${ }^{34}$. Ce 
profond questionnement entrepris par les féministes du monde de la culture, des principes fondamentaux de la théorie dramatique et de la performance pourrait avoir des conséquences importantes sur la conceptualisation du théâtre et sur les évaluations hiérarchiques de l'art.

La théorie différentialiste, qui soutient que les femmes et les hommes ont des différences biologiques qui résultent en une typologie de caractéristiques culturelles et une conscience corporelle distincte pour chaque sexe, pourrait aussi être significative pour la théorie du théâtre et de la performance et, par conséquent, pour la pensée historique ${ }^{35}$. Les généralisations sur la pensée « horizontale » plutôt que « linéaire » des femmes et la délimitation, génétiquement justifiée, d'une sphère sociale séparée pour les femmes, ont pu avoir un effet sur la façon dont les femmes ont fait du théâtre, et sur l'assertion d'une prérogative culturelle hégémonique des hommes à toutes les périodes de l'histoire. Dans la culture savante, les choix de motifs particuliers, de mythes, de légendes et d'événements historiques sont de la littérature dramatique. Le canon adopté de la littérature dramatique établit des rôles et une hiérarchie entre les spécialisations de métiers qui ont peu à voir ou n'ont rien à voir avec ce qui suscite la peur ou la crainte chez les femmes, ou avec ce que les femmes pourraient vivre à la place de la catharsis de la grande tragédie et de la chute fatale de personnages aristocratiques à qui la perspicacité, l'intuition et le bon sens commun font défaut. Ceci n'implique pas de point de vue marxiste provenant d'une culture jugée inférieure, ou d'une tradition alternative au sein d'une sous-culture oppressée et marginalisée. Cela ne requiert pas non plus de théorie psychanalytique sur les expériences spontanées de la femme générique.

Cependant, comme le souligne Gramsci, la forme imaginaire peut se transformer en véritable contenu : «Si le monde culturel pour lequel on lutte est un fait vivant et nécessaire [dans l'esprit de ses défenseurs], son expansivité sera irrépressible et il trouvera ses artistes ${ }^{36} \gg$. Cela est vrai du théâtre féministe de la seconde vague. Comme de nombreuses critiques littéraires féministes qui s'appuient sur le roman et la poésie, l'éclairage de Gramsci offre un point de départ pour interroger les traditions des formes théâtrales, la possession du pouvoir par ceux qui sont maîtres de la forme dominante, et l'appréciation de certains courants d'une alternative discrète, mais imaginative. L'histoire féministe a parcouru un long chemin depuis que Linda Nochlin avait tourné en dérision la question « Pourquoi n'y a-t-il pas eu de grands artistes 
femmes $^{37}$ ? » Les historien·ne.s du théâtre peuvent bénéficier des débats dans les beaux arts sur les traditions des pratiques artistiques, sur les appréciations culturelles des partisans du « génie artistique », et sur la réorientation d'une attention qui permet aux femmes de devenir visibles de leur plein droit, et pas seulement dans l'ombre des hommes.

\section{Vers une méthodologie féministe}

Les propositions suivantes pour une méthodologie féministe de l'histoire du théâtre ne préconisent pas de démarche technique ou mécanique, mais offrent un cadre théorique qui se traduit dans des principes organisationnels. Ces principes sont rassemblés dans trois questions de méthode, formulées de manière à ce qu'elles puissent s'appliquer aussi largement que possible, sans restriction à une période historique particulière.

Le résultat, je l'espère, pourrait mener à une recherche qui ne pourra pas être facilement divisée entre démarche « historique » ou « littéraire » parce qu'une alliance, quoique compliquée, a été réalisée entre ces deux pratiques complémentaires. Pour le moment, en attendant que d'autres recherches soient menées, beaucoup des remarques suivantes sont fatalement spéculatives. Elles sont destinées à consolider les contributions de recherches récentes (théoriques et études de cas approfondies) dans la théorie féministe et les études théâtrales, ainsi qu'à suggérer des hypothèses pour d'autres travaux en histoire du théâtre.

Chaque question se rapporte à des préoccupations professionnelles pour les personnes qui travaillent dans le théâtre. Il ne s'agit pas simplement d'étudier le phénomène des femmes travaillant dans le théâtre, ou d'identifier ce qui est irréfutablement «féministe » au théâtre, mais d'examiner les processus de travail et leur part de contrôle et de privilège par différents artistes et groupes sociaux, en s'efforçant d'en comprendre les implications sur le spectacle. Rendre visible ce qui a été invisibilisé dans l'expérience des femmes et des hommes est la voie de la connaissance de toute culture - non pas pour redresser un déséquilibre, mais parce que cette approche est plus rigoureuse et complète.

\section{Comment l'idéologie de la culture dominante affecte-t-elle le statut des femmes? \\ Les féministes socialistes mentionnent la séparation entre l'expérience genrée vécue au foyer (qui est privé) et sur le lieu de travail (qui est public). Cette séparation a été enfreinte à chaque fois qu'une actrice est montée sur une}


scène. La coexistence des actrices dans les deux sphères et leur construction sociale comme des « hommes honoraires » pour atténuer cette transgression est significative. Les femmes actrices (et dans une certaine mesure les femmes dramaturges et les techniciennes) personnifient la vie des femmes dans les limites du cadre de la société et de la culture masculines. Cela suggère qu’à certains égards les femmes de théâtre sont en meilleure posture que leurs homologues à l'extérieur du théâtre, mais généralement les jugements sociaux et l'ostracisme annulent ces avantages.

Une fois la participation des actrices dans la sphère publique officiellement approuvée par la loi et la société (en 1587 en Espagne et 1660 en Angleterre) ou par ailleurs permise par l'usage (certainement à partir des années 1530 en Italie et 1540 en France), elles ont gardé l'exclusivité des droits sur leurs professions (désignées par les termes féminisés de leur travail : actriz, balletgirl, comediante, cantatrice, etc.). Contrairement à la majorité des femmes, il était indispensable pour les actrices d'acquérir un apprentissage formalisé de leur métier - apprentissage qui incluait souvent certains arts libéraux : la musique, les langues et la grammaire - ce qui était approuvé et apprécié par la société qui les accueillait. Une fois qu'elles avaient fait leur place dans le milieu du théâtre, les actrices n’ont jamais empiété sur le marché du travail masculin ni affecté le barème des salaires des hommes dans le même métier ; elles étaient ainsi à l'abri de la pression de renoncer à leur gagne-pain pour laisser la place au pourvoyeur de la famille (masculin).

Un autre avantage, dans le passé, était qu'elles avaient de bonnes chances de recevoir salaire égal pour travail égal (tel qu'il était évalué par le succès du box-office) et semblaient jouir d'un accès, quoique restreint, aux plus hautes fonctions de direction, même si cela impliquait une compétence et expérience préalables. Un nombre impressionnant d'actrices victoriennes dirigèrent des théâtres, mais seulement quelques-unes d'entre elles réussirent à détenir un bail plus de deux ans ; celles qui bénéficiaient d'un bail à long terme étaient toujours associées à un partenaire masculin (comme Eliza Vincent et David Osbaldiston au Old Vic, Marie Wilton et Squire Bancroft au Prince of Wales Theatre), ou étaient parvenues à la direction de leur théâtre en tant que veuves (Sara Lane au Britannia, Ellen Poole au South London Palace of Varieties, et Mrs Nye Chart au Theatre Royal de Brighton). Dans d'autres cas, l'appartenance à une famille théâtrale a pu faciliter l'accès au bail - des recherches méritent d'être poursuivies, sur Louisa et Mary Ann Swanborough du Strand Theatre, ainsi que sur d'innombrables directrices 
dont les biographies ne figurent pas dans les Who's Whos contemporains ou les rubriques nécrologiques du théâtre, et ont ainsi échappé au guide de J.P. Wearing sur les sources biographiques du monde du théâtre ${ }^{38}$.

En exerçant une profession précaire, itinérante et bohème, les femmes interprètes ont repoussé la conscience traditionnelle des femmes axée autour du foyer, et se sont engagées dans une lutte active contre l'idéologie de la culture (masculine) dominante. Quand la religion officielle et les institutions légales de la culture masculine n'empêchèrent pas formellement les femmes d'apparaître sur scène, le contexte culturel définissait quant à lui la signification sociale de leur vie publique en tant qu'actrices, et fixait le plus souvent chaque aspect de leur statut pour la communauté entière sans tenir compte des comportements individuels effectifs. Le lieu de travail des actrices, qui était aussi un lieu d'activité sexuelle, impliquait qu'elles bénéficiaient de libertés inconnues des femmes qui exerçaient d’autres métiers. [Les actrices] étaient le symbole de l'autonomie et de l'indépendance des femmes, mais bien que leur indépendance, leur éducation, leur attrait, et le fait qu'elles enfreignent les bonnes mœurs sexuelles de leur société leur aient donné accès à l'élite dirigeante masculine, cela représentait également une dangereuse transgression du tabou lié à leur genre, suffisamment semblable à de la prostitution pour que des objections soient formulées contre elles.

Le statut ambigu des actrices sous la Restauration comme Hester Davenport, Elizabeth Barry et Peg Hughes, provenait de leurs liaisons sexuelles qui, à leur tour, résultaient de leur mise à disposition comme commodité érotique ${ }^{39}$. Tout comme les prostituées, les actrices étaient, en public, quotidiennement confrontées à ce deux poids deux mesures. Elles passaient outre les structures familiales traditionnelles, l'équilibre du pouvoir économique, et les restrictions liées au genre dans la création d'associations professionnelles, dans leur liberté de circulation, l'habillement, l'éducation, l'influence et les choix sexuels. Leur expatriation sexuelle de la sphère domestique, le défi à la suprématie patriarcale dans la sphère publique, et le fait de troubler les prérogatives des hommes dans la sphère sexuelle ont souvent conduit à leur ostracisme et diffamation ${ }^{40}$. Tandis qu'elles auraient pu incarner le prototype des héroïnes féministes, leur indépendance, leur courage et leur suprématie se sont amèrement transformés en dépendance, compromis, et survie à peine acceptable. 
Comment les critères sociaux, de classe, et économiques, affectent-ils les privilèges?

Le théâtre ayant été traditionnellement une entreprise familiale, il semble tout à fait logique que les liens de parenté (ou le manque de liens de parenté) aient affecté, les possibilités pour les actrices d'accéder à la scène, leurs chances de réussite, leurs rémunérations financières, leurs débouchés sur des postes de gestion, leur sécurité d'emploi ${ }^{41}$. Bien que le noyau familial n'ait que rarement délimité la réelle unité de production, le théâtre est parvenu à maintenir ensemble les domaines de la production et de la reproduction plus longtemps et de manière plus équitable que bien d'autres secteurs de l'économie après l'industrialisation. Qu'il s'agisse du système de parts courant à la Renaissance ou du travail rémunéré du dix-neuvième siècle, les emplois pour chacun des membres de la famille - indépendamment du sexe, de l'âge et des compétences - étaient construits selon les traditions des dramatis personae, qui comprenaient l'apprentissage du métier d'acteur. $\mathrm{Au}$ cours du temps, parmi les Andreinis, Kembles et Terry-Lewises, le salaire du noyau familial ou de la famille élargie était collectivement gagné par le travail de tous les membres ou bien seulement grâce à la célébrité exceptionnelle de quelques-un.e.s d'entre eux ou elles, et tou.te.s en tiraient des avantages. Par conséquent, dans les biographies, l'implication des femmes dans la famille élargie et leurs rôles dans le noyau familial (qu'il s'agisse du mariage, de la maternité et de l'éducation des enfants - selon les cas de figures) nécessitent d'être étudiée en lien avec leur apprentissage en tant qu'actrice, l'avancement de leur carrière, leur retraite, leur réseau de relations professionnelles et familiales.

Tout comme les femmes, les praticien.ne.s du théâtre ne formaient pas une unique classe économique ou sociale ${ }^{42}$. Constat moins évident : contrairement à la plupart des femmes, la classe économique des actrices était rarement conforme à leur statut social. Quelle que soit la réussite financière d'une danseuse romaine ou d'une Gaiety Girl victorienne [danseuse interprète du chœur des comédies musicales du Gaiety Theatre de Londres, célébrées dans les années 1890], ses revenus étaient toujours considérés comme provenant de sources illégitimes et elle demeurait une demi-mondaine. La renommée qui pouvait être acquise à travers la spécialisation dans un domaine « sérieux » (particulièrement la tragédie) renforçait la crédibilité sociale et tendait à réduire l'écart entre le salaire et le statut; même si les comédiennes et les acrobates pouvaient être tout aussi reconnues parmi les 
professionnel.le.s et être aussi bien payées que les tragédiennes, elles ne pouvaient espérer atteindre la même reconnaissance sociale. Et la société, et le monde professionnel établissaient une hiérarchie entre interprètes, mais leurs critères de hiérarchie n'étaient pas toujours identiques.

L'idée que l'affectation à une classe est une condition à vie est également inappropriée en ce qui concerne les actrices. Le mariage à l'intérieur ou à l'extérieur de la profession pouvait radicalement changer le rang d'une femme (et même faire d'elle une impératrice, dans le cas de Theodora) et une rupture avec ses origines bourgeoises ou avec un milieu protestant strict pouvait déshériter une aspirante à la scène (comme ce fut le cas pour Carolina Neuber) qui, en tant que femme, n’a pas réintégré les lois de la primogéniture. Les indicateurs socio-économiques des privilèges des actrices, qui reposent sur la propriété et le contrôle des biens sont des repères quantitatifs qui renseignent sur le rang social de l'actrice sans être entièrement fiables : l'analyse se complexifie par la pratique du port de bijoux (loués pour l'occasion ou en pâte de verre), par la parenté (la part immobilière ou financière d'une personne - et particulièrement d'une femme - dans les biens et capitaux collectifs est difficile à estimer) et par les ascensions professionnelles vers des postes de direction réservés aux veuves, mais peu accessibles aux épouses (transaction faisant partie de l'héritage habituel des music-halls victoriens de taille modeste $)^{43}$. Le statut artistique et social des interprètes femmes était en partie déterminé par leur accès à l'éducation, les types d'emplois et la fréquence des embauches, et les biens propres, mais les relations sexuelles et familiales étaient généralement liées - ouvertement ou secrètement - à leur statut.

\section{De quelle façon le statu quo est-il maintenu ou contesté dans les formes artistiques?}

Les féministes considèrent la littérature comme une institution sociale : le théâtre place la littérature au sein d'une autre institution sociale où des spectateurs éprouvent, dans un acte communautaire, le plaisir partagé de voir et d'entendre. Nancy Reinhardt décrit le théâtre comme un art conservateur et avance que sa forme et son contenu conservateurs peuvent rendre son sexisme moins flagrant que dans un média comme le cinéma. Si cela est véridique, le rôle culturel du théâtre comme transmetteur d'images et producteur d'images dans l'élaboration des représentations sociales est particulièrement lié à l'idéologie de la culture dominante et particulièrement susceptible de la renforcer. La participation des femmes à cette sphère professionnelle 
peu conventionnelle peut être interprétée comme le prolongement naturel du traitement légal, médical et artistique de la femme comme entité, ce qui explique que les figures de femmes soient pensées dans un ensemble d'antithèses, dont aucune n'est positive : ainsi les femmes sont rabaissées ou divinisées, de sauvages êtres sexuels ou de moribondes reproductrices, mentalement superficielles ou intellectuellement subversives, femmes d'apparat ou hideuses, socialement périphériques ou très dangereuses. Tout comme les praticien.ne.s du théâtre ont tendance à considérer la création théâtrale comme un processus collectif impliquant différents savoir-faire, les historien.ne.s ont tendance à considérer l'activité de spectateur comme fédératrice d'une communauté spécifique, mais la prétendue « neutralité » des producteurs d'images et des images produites requiert un examen minutieux. Les historien.ne.s devraient examiner à quel point la création et la réception sont vraiment d'ordre « communautaire » dans une culture donnée : peut-être qu'en réalité, le conservatisme dissimule un sexisme plus (et non pas moins) flagrant.

Ce sexisme est douloureusement évident pour les femmes qui travaillent dans le théâtre. Aujourd'hui, la concentration de l'emploi des femmes dans les théâtres régionaux, les théâtres jeune public, l'éducation artistique et théâtrale, et les relations publiques, tient probablement plus à la pratique professionnelle et institutionnalisée du sexisme qu'à une inclination personnelle. Michelene Wandor présente un éventail de réponses de femmes face à des conditions de travail multiples :

Une féministe travaillant dans une création théâtrale traditionnelle, où les attitudes sont particulièrement réactionnaires, est susceptible de ressentir des colères féministes radicales, fortes, viscérales; une femme en position de pouvoir... doit affronter et gérer ses désirs de pouvoir et son pouvoir effectif au travail. De plus, une féministe qui est en mesure de travailler démocratiquement avec les autres... est davantage susceptible de chercher à explorer les façons de partager le pouvoir et la conduite du travail avec d'autres, tout en assumant ses responsabilités et son travail en tant que femme ${ }^{44}$.

Tous ces scénarios ont été décrits dans les témoignages d'artistes comme Liane Aukin et Ann Jellicoe [Liane Aukin (1936-2016) et Ann Jellicoe (19272017) : autrices, actrices et metteuses en scène anglaises $]^{45}$. Les conséquences sur les carrières des femmes ont également été observées par Chinoy, qui constate que le choix de la dignité et de la démocratisation au travail par et pour 
les travailleurs et les travailleuses, a conduit les femmes de théâtre américaines à s'associer à ou à fonder des théâtres régionaux, institutionnels, locaux, des théâtres d'art ou alternatifs, plutôt que de passer leur vie à essayer de se faire une place à Broadway ou sur la scène commerciale ${ }^{46}$. Selon Chinoy, le travail d'Eva Le Gallienne, Susan Glaspell, Minnie Maddern Fiske et Hallie Flanagan Davis prend des résonances particulières parce qu'elles étaient des femmes. Une autre interprétation de leur parcours professionnel (parallèlement au travail de Joan Holden, Ellen Stewart, Megan Terry, Joan Littlewood et peutêtre Eliza Vestris) est qu'en tant que femmes qui occupaient des postes où elles prenaient des décisions artistiques, financières ou administratives, elles inversaient les structures habituelles du pouvoir aussi bien dans la société qu'au théâtre. À moins qu'elles ne soient ségrégationnistes et ne souhaitent travailler qu'entre elles, ces femmes doivent exiger que le pouvoir leur soit attribué par les interprètes et les technicien.ne-s qui sont accoutumés à l'autorité masculine ; ceci est plus simplement réalisable et gratifiant lorsque les concernées opèrent dans des structures alternatives plus petites. Les femmes ont, sans nul doute, eu et continuent d'avoir une incidence sur l'administration des théâtres. En effet, les recherches empiriques montrent que les femmes qui sont à des postes de direction tendent à recruter femmes et hommes de manière plus équitable ${ }^{47}$. Il reste à démontrer cependant si la réalité sociale et les expériences vécues parviennent à être présentes dans les spectacles de compagnies mixtes (contrairement au Projet Magdalena) qui leur sont favorables, et jusqu'aux spectateurs et spectatrices, dans un public mixte (contrairement à quelques-uns des exemples de Leavitt ${ }^{48}$ ), ce qui contesterait le statu quo à tous les niveaux.

Pour les historien.ne.s des pratiques artistiques et culturelles, la question des statu quo est essentielle pour comprendre la façon dont les idées relatives à la sexualité sont relayées ou revisitées socialement. Tandis que la littérature est une activité personnelle de l'imagination (pour l'écrivain et le lecteur ou la lectrice), le théâtre a lieu dans le temps présent (de manière éphémère) sous les yeux du public. Pour qu'il y ait théâtre, il doit y avoir des témoins ; leur réaction fait autorité sur les critiques qui émanent d'une élite culturelle ou sur les rapports que les historien.ne.s rédigent a posteriori.

Le théâtre communiqueà travers des moyens hautementreprésentationnels, dans les systèmes extrêmement codifiés, non seulement du langage, mais aussi du discours, non seulement de configuration, mais aussi d'action, 
non seulement d'événements sélectionnés, mais aussi d'événements mis en lumière, costumés, agencés et joués - dans certains cas, maintes et maintes fois. Un texte porté à la scène grâce aux ressources de l'expression scénique et reçu de manière empirique par le public à l'occasion de sa présentation devrait être appelé une mise en scène [en français dans le texte]. S'il était possible d'《écrire » la partition d'un spectacle de théâtre de la même façon que la musique est notée, et de prendre en compte tous les composants de l'expression scénique (costumes, décors, musiques, lumières, éléments scénographiques et accessoires, actions, mots, lignes, couleurs, intonations, rythmes, etc.), l'expérience de lire la mise-en-scène pourrait être la même que l'expérience de faire partie du public. Mais cela n'est pas possible car, aussi complète soit la partition, ce n'est qu'un rendu partiel d'une expérience réelle et immédiate. Les rapports de genre, et leur codification élaborée sont inséparables de la mise en scène (et essentiels à la vraisemblance ou l'invraisemblance du spectacle). En tant qu'élément lisible des conventions sociales, le genre est infiniment sujet à redéfinition ${ }^{49}$.

La mise-en-scène est affectée par les choix de distribution des rôles, de costumes, d'usage de l'espace selon la configuration narrative, de décor et d'actes de langage ; les valeurs sociales attribuées au genre peuvent changer la signification apparente de chacun de ces éléments, et vice versa. Le travestissement fournit un exemple intéressant de la façon dont les valeurs sociales sont présentes dans la distribution traditionnelle des rôles, et de la façon dont les costumes et gestes forment une réalité théâtrale genrée. Quand, par exemple, les femmes étaient bannies, par la loi ou les coutumes, des scènes occidentales et orientales, les hommes jouaient les rôles de femmes ${ }^{50}$. Le comportement adopté par un acteur selon son expression personnelle de la « féminité » influence la réception, qui, à la Renaissance, a pu être entremêlée à l'homo-érotisme des spectateurs hommes et au narcissisme des femmes spectatrices $^{51}$. La capacité de l'audience à interpréter ce transvestisme était déterminée par des conventions liées au sens ; plus qu'un style de jeu était impliqué dans ces expressions du genre.

Dès lors, les historien.ne.s féministes peuvent se demander : quand les femmes étaient exclues du forum public de la scène théâtrale et que les hommes jouaient les rôles de femmes, était-ce nécessairement un acte d'anéantissement des femmes, d'usurpation de l'existence des femmes, de misogynie, de réduction au silence ou de moquerie ? Les gestes, les mots 
et les robes des femmes ont-ils été repris par les hommes pour railler ces dernières ? Était-ce identique au grimage en noir (comme dans les minstrelsy, [spectacles apparus aux États-Unis à la fin des années 1820, dans lesquels des acteurs blancs se noircissaient le visage en noir pour interpréter des Noirs béats et souvent idiots, doués pour la musique et la danse]) ? Sue-Ellen Case fait de l'invention mythique du jeu de l'acteur une construction masculine : Arion donnait aux chanteurs (hommes) des dithyrambes les costumes des satyres (hommes).

Le pouvoir de la représentation n'a été donné qu'aux célébrants hommes. L'invention du jeu de l'acteur était rattachée à un genre particulier - l'acteur était le satyre. La spécificité du genre de l'acteur dans les pièces satiriques était soulignée par le port d'un phallus de cuir. Cependant, afin de dramatiser le combat entre les genres, la femme devait être représentée d'une manière ou d'une autre... Dans l'étude de cette représentation, il est important de rappeler que la notion du féminin provenait du point de vue masculin, qui restait étranger à l'expérience des femmes et reflétait la perspective de son opposé en termes de genre. Ce vocabulaire de gestes a inaugurél'image de la « Femme », telle qu'elle est vue sur scène - qu'elle s'est institutionnalisée à travers la culture patriarcale et qu'elle a été représentée par des signes, façonnés par des hommes, du comportement qui était attendu d'elle ${ }^{52}$.

Si, comme Case le montre avec à l'appui la Poétique et la Politique d'Aristote, l'institutionnalisation était anti-femme, que pouvait signifier, quand par la suite les femmes ont été admises sur scène, de jouer ces mêmes textes écrits pour être interprétés par des hommes ? Ont-elles continué à se noircir, ou se sont-elles blanchies ? Quand les mots, les robes, les gestes et le genre deviennent-ils ceux des personnages féminins et des interprètes femmes ? Demeurent-ils masculins, même quand ils sont prononcés par des femmes? Indépendamment du genre de l'interprète initial, le jeu d'acteur est-il un art masculin - immuablement habillé en costume du satyre - et peut-il être féminisé ?

Depuis la Restauration, les conventions autorisent les femmes à se travestir - une femme peut interpréter un rôle d'homme - libération jusquà une quasi-masculinité qui autorise son personnage à faire des choses et à ne pas être un simple vilain, une harpie ou un aventurier sexuel. Elle pouvait prendre des initiatives romantiques (en étant Roméo, ou un jeune pantomime), des 
libertés comiques (en étant Falstaff, ou dans le burlesque), ou même faire la satire des hommes et de la masculinité (comme un Hercule de musichall). Ceci n'est pas une évolution entièrement positive : l'identité sexuelle de l'actrice travestie n'a jamais été ambiguë ; le travestissement a souvent été utilisé pour dévoiler sa silhouette et entourer sa présence d'érotisme, faisant d'elle une exhibitionniste soumise à l'examen du public masculin. Les récompenses érotiques du spectateur quand il regarde Charlotte Cushman interpréter Roméo ou Madame Webb dans le rôle de Falstaff peuvent avoir été minimes ${ }^{53}$, mais le procédé est établi par Peg Woffington, Dora Jordan, Charlotte Clarke, Priscilla Horton et Fanny Wallack [actrices irlandaises ou anglaises des $\mathrm{XVIII}^{\mathrm{e}}$ et $\mathrm{XIX}^{\mathrm{e}}$ siècles, célèbres de leur temps, pour leurs interprétations de rôles de femmes travesties en hommes dans les pièces de Shakespeare notamment $]^{54}$. L'actrice travestie n'avait pas la possibilité de se faire passer pour l'autre sexe ; elle ne pouvait qu'indiquer le sien. L'acte politique de son travestissement est foncièrement différent de celui du jeune garçon-actrice, car dans son cas, la masculinité n'a pas été substituée par la féminité.

L'usage de l'espace scénique et son rôle dans l'intrigue mise en scène ont été compris comme des éléments relevant du genre. Les historien.ne·s féministes ont montré que le positionnement des personnages sur scène (par des dramaturges et régisseurs/metteurs en scène) est porteur de sens. Selon Reinhardt, le centre de la scène est le lieu des protagonistes masculins, tandis que « les côtés, le fond de scène, les niches et les balcons fonctionnent comme un espace intérieur domestique où les femmes sont généralement maintenues... Si, en tant que personnage sur scène, [la femme] défie les conventions et envahit le centre de la scène, elle est souvent exagérée ou déformée comme l'est "un ange ou un monstre" $\$ 5$ ». Dans ce cas, l'image scénique matérialise la division sociale de deux sphères et toute femme qui transgresse est aussitôt reconnaissable par sa position sur scène. Jenkins montre que le décor joue un rôle similaire :

[Dans des pièces de théâtre écrites par des hommes] les hommes nomment et revendiquent l'usage de l'espace et du territoire. Notez que les hommes revendiquent l'usage de certains espaces dans la sphère domestique. Ils ont une place d'honneur dans le salon et la salle à manger... Ils ont même leurs propres bureaux et ateliers. Alors que la cuisine est la sphère de la femme, les hommes ont leur place à la table et ont accès à tout ce qui se trouve dans la cuisine (qui a accès à son bureau, son atelier? ?) ${ }^{56}$. 
Ces images de rôles sociaux sont perçues par le public, du moins inconsciemment. La culture occidentale interprète le centre comme le pouvoir, et la périphérie comme le silence. Elle interprète le confinement comme un emprisonnement pour les hommes et une socialisation adéquate pour les femmes.

Il est volontiers admis que l'attribution de mots par les dramaturges aux personnages reflète les équilibres de pouvoirs en jeu dans le drame, aussi bien que les rapports de force dans la troupe de théâtre. La capacité des interprètes à bénéficier de privilèges dépendait de leur accès à l'éducation, à la culture artistique, au capital et au mécénat ; pour les hommes, il y avait les clubs sociaux, les auberges publiques, les sociétés secrètes et les partis politiques pour se réunir entre alliés, mais pour les femmes, la sexualité était prééminente. À l'exception de rares actrices, se soumettre au système d'exposition de soi et d'attraction était nécessaire à l'avancement professionnel. Le théâtre a, à sa disposition, deux moyens principaux d'attirer, les mots et les images, l'un et l'autre se retournant contre les actrices. Tandis que le monologue est considéré comme de l'éloquence chez les hommes, il est habituellement reçu comme un signe de verbosité chez les femmes. Alors qu'un costume ostentatoire indique généralement la détention de pouvoir chez les hommes, il est le signe d'autopromotion sexuelle chez les femmes, que cela fasse sens ou non avec la fiction dramatique, soit conforme ou non à la mise en scène.

Enfin, les femmes spectatrices ont joué un rôle dans le maintien du statu quo. Si les femmes étaient moins nombreuses à assister aux spectacles que les hommes dans une période donnée, comment se fait-il qu'elles s'en soient maintenues à distance ? Que cela peut-il indiquer au sujet de la société, du quartier aux alentours du théâtre, de la signification sociale de l'événement théâtral, de l'hégémonie masculine sur la culture, de la distribution des revenus, et des habitudes de dépenses? Dagmar Höher a découvert que, tandis que les femmes qui n'avaient qu'un seul enfant continuaient à fréquenter le music-hall, deux enfants ou plus les retenaient à la maison, ce qui diminuait le revenu de la famille, relégué le loisir de la fréquentation des music-halls à une prérogative masculine jusqu'à ce que les enfants grandissent, que les dépenses du ménage baissent, et que les femmes reprennent leur plaisir de jeunesse ${ }^{57}$ ? L'importance du mécénat de classe et de sa réflexion sur et autour du répertoire est largement reconnue, mais l'interdépendance entre le répertoire et, la classe et le genre des spectateurs, constitue un champ 
de recherche prometteur. De même, analyser la fréquentation des salles de spectacles par les femmes en lien avec la dimension sociale ou de parenté, pour chacune des périodes de l'histoire, $\mathrm{y}$ compris la période actuelle, soulève des questions féministes qui supposent d'observer les liens culturels, les rites sexuels, la sécurité publique des femmes et la redéfinition continuelle de la femme à travers le biais du microcosme théâtral de la société.

Bien qu'il y ait beaucoup à apprendre de l'histoire féministe et sociale récente, la façon dont l'historien·ne traite l'événement théâtral qui n'est réel qu'à certains niveaux, suggère que l'histoire du théâtre requiert l'usage d'outils analytiques et de sources primaires qui ne sont pas utilisés par les histoires non artistiques. La nature éclectique du théâtre (et par conséquent de l'histoire du théâtre) est complétée par une approche plurielle de l'histoire féministe qui cherche constamment à interroger les traditions par lesquelles le savoir est reconnu, rejetant souvent à la fois les traditions et le savoir ainsi produits. En délimitant une nouvelle série de questions à prendre en considération dans la fabrique de l'histoire, les recherches féministes peuvent exploiter de nouveaux types de sources, élargir la base intellectuelle et identifier les données jusqu'ici ignorées ou sous-évaluées, ainsi que réexaminer le familier. Comme l'affirme Lynda Nead, «le féminisme redéfinit les objets et les objectifs des recherches... et transforme la discipline elle-même ${ }^{58} \gg$. Pour ce faire, tout ce qui porte sur le fonctionnement de la différence de genre et sur la sexualité dans le théâtre constitue un travail nécessaire.

\section{Notes}

1. Gerda Lerner, The Majority Finds its Past, New York, Oxford, Oxford University Press, 1979, p. 168-180 ; et Teaching Women's History, Washington D.C., American Historical Association, 1981.

2. Michelene Wandor, parmi les critiques, soutient qu' " une approche dualiste nie et verrouille toute forme de discussion ", dans Look Back in Gender, London, New York, Methuen, 1985, p. xvi.

3. Un ensemble de contributions représentatives sont réunies dans Elaine Showalter, ed., The New Feminist Criticism : Essays on Women, Literature, and Theory, London, Virago, 1986 ; et Gayle Green et Coppelia Kahn, eds., Making a difference : feminist literary criticism, London et New York, Methuen, 1985. L'état des lieux critiques de Toril Moi des tendances de la critique féministe est utile et riche d'enseignements, mais ne prend en compte ni l'art dramatique, ni le théâtre. Sexual Textual Politics: Feminist Literary Theory, London et New York, Methuen, 1985. Note de la traductrice : le terme de 
gynocritique, forgé par Elaine Showalter en 1979, est utilisé au Canada. Il propose un cadre d'analyse propre aux textes produits par les femmes autrices pour se libérer des modèles de critiques masculins, et tend à définir une spécificité de l'écriture au féminin.

4. Janelle Reinelt, «Beyond Brecht: Britain's new feminist drama », Theatre Journal, vol. 38, n² 2, mai 1986, p. 154-163, et Phyllis Rackin, « Androgyny, mimesis, and the marriage of the boy heroine on the English Renaissance Stage », PMLA (Journal of the Modern Language Association of America), vol. 102, n 1, janvier 1987, p. 29-41.

5. Erik Aschengreen "The Beautiful danger : facets of the Romantic ballet ", Dance Perspectives, n 58, été 1974.

6. Ann Daly, "The Balanchine Woman: Of Hummingbirds and Channel Swimmers ", The Drama Review, vol. 31, n 1, printemps 1987, p. 8-21.

7. Jill Dolan, «The Dynamics of Desire: Sexuality and Gender in Pornography and Performance », Theatre Journal, vol. 39, n 2, mai 1987, p. 156-174.

8. Tracy C. Davis, "Sexual Language in Victorian Society and Theatre ", American Journal of Semiotics, $6: 1,1989$.

9. Nancy S. Reinhardt, «New directions for feminist criticism in theatre and the related arts ", dans A Feminist perspective in the academy: the difference it makes, ed. Elizabeth Langland and Walter Gove, Chicago, University of Chicago Press, 1981, p. 25-26 ; et Claudia D. Johnson, «That guilty third tier: Prostitution in the nineteenth-century American theaters ", dans Victorian America, ed. David Walker Howe, Philadelphia, University of Pennsylvania Press, 1976, p. 111-120.

10. Sheila Rowbotham, "The trouble with patriarchy " dans People's history and Social theory, ed. Raphael Samuel, Londres, Routledge and Kegan Paul, 1981, p. 367, 366.

11. J. S. Bratton, "Jenny Hill: sex and sexism in the Victorian music hall ", dans Music hall performance and style, ed. J. S. Bratton, Milton Keynes, Open University Press, 1986, p. 103.

12. Ibid.

13. Rina Fraticelli, "The invisibility factor: Status of women in Canadian theatre ", Fuse, vol. $6, n^{\circ} 3$, septembre 1982, p. 112-124. [Note de la traductrice : Une version française de cet article a été publiée dans Jeu, n ${ }^{\circ} 31,1984$, p. 65-107, Consulté le $1^{\text {er }}$ février 2018. URL : https://www.erudit.org/fr/revues/jeu/1984-n31-jeu1065879/28459ac.pdf]; Kar Carney et Julianne Boyd, «Directors and designers report on sex discrimination in the theatre ", Women and Performance, vol. 1, n², hiver 1982, p. 46-54.

14. Judith Lynne Hanna, "Patterns of dominance: men, women, and homosexuality in Dance », The Drama Review, vol. 31, n 1, t. 113, printemps 1987, p. 23.

15. Rosamond Gilder, Enter the Actress: the First Women in the Theatre, 1931, rpt. New York, Theatre Art Books, 1960 ; Albert Auster, Actresses and Suffragists: Women in American Theatre, 1890-1920, New York, Praeger, 1984 ; Claudia D. Johnson, American Actress: Perspective on the Nineteenth Century, Chicago, Nelson-Hall, 1984 ; et Katharina M. Wilson, ed., Medieval Women Writers, Manchester, Manchester University Press, 1984.

16. Sherry B. Ortner et Harriet Whitehead, eds., Sexual Meanings: the Cultural Construction of Gender and Sexuality, Cambridge, Cambridge University Press, 1981.

17. Susan Dwyer Amussen, «Féminin/ masculin : le genre dans l'Angleterre de l'époque moderne », Annales. Économies, Sociétés, Civilisations, Année 1985, vol. 40, n 2, p. 269-287. 
18. Claire Hirshfield, «The Actresses' Franchise League and the campaign for women's suffrage 1908-1914 », Theatre Research International, vol. 10, n² 2, été 1985, p. 129-153; et Julie Holledge, Innocent Flowers: Women in the Edwardian Theatre, Londres, Virago, 1981 ; Charlotte Rea, «Women's theatre groups », The Drama Review, vol. 16, n 2, juin 1972, p. 79-89, Catherine Itzin, Stages in the Revolution: Political Theatre in Britain since 1968, Londres, Methuen, 1980 ; Dinah Luise Leavitt, Feminist Theatre Groups, Jefferson, N.C, McFarland, 1980 ; Marie-Claire Rouyer et Anne Cipriani, «Women's theatre in Great Britain ", Fireweed, 7, été 1980, p. 46-61 ; et Susan E. Bassnett, "Towards a theory of women's theatre ", dans Semiotics of Drama and Theatre, ed. Herta Schmid and Aloysius Van Kesteren, Amsterdam et Philadelphie, John Benjamins, 1984, p. 445-466.

19. Fidelis Morgan, The Female Wits, Londres, Virago, 1981 ; Dale Spender et Carole Hyman, eds, « How the Vote Was Won » and Other Suffragette Plays, Londres et New York, Methuen, 1985 ; Viv Gardner, ed., Sketches from the Actresses' Franchise League, Nottingham, Nottingham Drama Texts, 1985 ; et C. W. E. Bigsby, ed., Susan Glaspell, Cambridge, Cambridge University Press, 1987 ; Michele Wandor, Look Back in Gender, New York, Methuen, 1987 ; Honor Moore, ed., The New Women's Theatre: Ten Plays by Contemporary American Women, New York, Vintage, 1977 ; Julia Miles, ed., The Women's Project, 2 vols., New York, Performing Arts Journal and American Place Theatre, 1980 et 1984 ; Judith E. Barlow, ed., Plays by American Women: 1900-1930, New York, Applause, 1981 ; Michelene Wandor, ed., "Strike While the Iron is Hot »: Three Plays on Sexual Politics, Londres et West Nyack, Journeyman Press, 1980 ; Michelene Wandor, ed. Plays by women, 6 vols., Londres, Methuen, 1982, 1983, 1984, 1985, 1987, 1988 ; and Kate McDermott, ed. Places, Please! The First Anthology of Lesbian Plays, Iowa City, Aunt Lute, 1985.

20. Lynda Nead, «Feminism, art history and cutural politics ", dans The New Art History, ed. A. L. Rees and Frances Borzello, Londres, Camden Press, 1986, p. 120-124.

21. Nancy Cotton, Women Playwrights in England c. 1363-1750, Lewisburg, Bucknell University Press, 1980.

22. Note de la traductrice : Thomas Postlewait, historien de renommée internationale éditeur notamment de The Cambridge Introduction to Theatre Historiography en 2009 et plus récemment avec Charlotte Cunning de Representing the Teatrical Past: Essays in the Historiography of Performance, University of Iowa Press, 2011, co-édite avec Bruce A. McConachie, Interpreting the Theatrical Past, Essays in the Historiography of Performance où est publié le présent article de Tracy Davis. Thomas Postlewait intitule sa contribution à ce même recueil « Autobiography and theatre history », p. 248-272.

23. Marie-Claire Pasquier, "Women in the theatre of men: What price freedom? ", dans Women in Culture and Politics: a Century of Change, ed. Judith Freidlander et al., Bloomington, Indiana University Press, 1986, p. 194-206.

24. Linda Walsh Jenkins, Women in American Theatre: Careers, Images, Movements. An Illustrated Anthology and Sourcebook, ed. Helen Crich Chinoy and Linda Walsh Jenkins, New York, Crown, 1981, p. 237.

25. Roberta Sklar, "Toward creating a women's theatre ", entretien de Cornelia Brunner, The Drama Review, vol. 24, $\mathrm{n}^{\circ}$ 2, Women and Performance Issue, juin 1980, p. 23-40. Linda Walsh Jenkins, "Locating the gender experience ", Women and Performance, vol. 2, n¹, p. 5-17 ; et Linda Walsh Jenkins et Susan Ogden-Malouf, «The (female) 
actor prepares », Theatre, vol. 17, $\mathrm{n}^{\circ} 1$, hiver 1985, p. 66-69. Note de la traductrice : Nous traduisons " maleness ", non par masculinité mais par " masculiniste » empruntant le terme à Michèle Le Doeuff, dans L'Étude et le Rouet, tome 1, Des femmes, de la philosophie, etc., Paris, Seuil, p. 55 : «Pour nommer ce particularisme, qui non seulement n'envisage que l'histoire ou la vie sociale des hommes, mais encore double cette limitation d'une affirmation (il n'y a qu'eux qui comptent, et leur point de vue), j'ai forgé le terme masculinisme ».

26. Vesna Pistotnik, «Towards a redefinition of dramatic genre and stage history », Modern Drama, vol. 28, n 4, décembre 1985, p. 681.

27. Kathleen Betsko et Rachel Koening, eds., Interviews With Contemporary Women Playwrights, New York, William Morrow, 1987.

28. Note de la traductrice : Institution éducative indépendante d'Oxford, soutenue par les syndicats, fondée en 1889 par John Ruskin qui offre une éducation supérieure à moindre coût à des travailleurs et travailleuses qui avaient quitté l'école sans qualifications pour l'université.

29. Note de la traductrice : Le gérondif « silencing» désigne l'action en train de se faire, les modalités et processus de cette mise sous silence.

30. Rachel Koening, op. cit., «Introduction », Interviews, p. 12.

31. Elisabeth J. Natalle y parvient en insistant sur la fonction rhétorique d'œuvres récentes produites par des femmes, dans Feminist Theatre: a Study in Persuasion, Metuchen, N.J., et Londres, Scarecrown Press, 1985.

32. Lisa Jardine, Still Harping on Daughters: Women and Drama in the Age of Shakespeare, Brighton, Sus. : Harvester press ; Totowa, N.J. : Barnes and Noble books, 1983.

33. Carol W. Gelderman, «The Male Nature of Tragedy », Prairie Schooner, Automne 1975, p. 220-236 ; Leavitt, Feminist Theatre Groups ; Karen Malpede, ed., Women in Theatre: Compassion and Hope, New York, Limelight, 1983 ; The Drama Review, vol. 24, n 2, juin 1980 ; et la Canadian Theatre Review, 43, été 1985.

34. Susan Bassnett, "Women Experiment with Theatre: Magdalena 86 », New Theatre Quarterly, vol. 3, n 1, août 1987, p. 224-234.

35. Ceci est nié par Eugenio Barba, "The Actor's energy: male/female versus animus/ anima ", New Theatre Quarterly vol. 3, n 1, août 1987, p. 237-240, qui insiste sur le fait que la pré-expressivité est asexuelle, postulat discuté par Bassnett, «Perceptions of the Female Role: The ISTA Congress ", p. 234-237 dans la même revue.

36. Antonio Gramsci, Selections from cultural writings, ed. David Frogacs and Geoffrey Nowell-Smith, Cambridge, Mass, Harvard University Press, 1935, p. 109. Traduction française issue de Problèmes de civilisation et de culture, "Critères de critiques littéraires » (1933), consulté le 2 février 2018, URL : https://www.marxists.org/francais/ gramsci/intell/intell3.htm (trad. non mentionné e).

37. Linda Nochlin, "Why have there been no great women artists? ", dans Art and Sexual Politics: Women's Liberation, Women Artists, and Art History, ed. Thomas B. Hess et Elizabeth C. Baker, New York, Macmillan, Londres, Collier Macmillan, 1973. Note de la traductrice : l'article et l'ouvrage de Nochlin a été traduit en français par Oristelle Bonis, Femmes, art et pouvoir, et autres essais, Nîmes, Chambon, 1993. 
38. Voir Diana Howard, London Theatres and Music Halls, 1850-1950, London, Library Association, 1970 ; et J.P. Wearing, American and British Theatrical Biography: a Directory, Metuchen, N.J., et Londres, Scarecrow, 1979.

39. Antonia Fraser, «The actress as honey-pot ", The Weaker Vessel, Londres, Methuen, 1984, p. 473-496.

40. Tracy C. Davis, «Does the theatre make for good?: actresses' purity in the Victorian era », Queen's Quarterly, vol. 93, n¹, printemps 1986, p. 33-49.

41. Michael Baker, The Rise of the Victorian actor, Londres, Croom Helm, 1978, p. 62-94.

42. Joan Kelly, Women, History, and Theory: the Essays of Joan Kelly, Chicago, University of Chicago Press, 1984, p. 5.

43. Diana Howard, London theatres and music halls, 1850-1950, op. cit.

44. Michelene Wandor, Carry on, Understudies: Theatre and Sexual Politics, $2^{\text {nd }}$ édition, Londres, Routledge et Kegan Paul, 1985, p. 138.

45. Susan Todd, ed., Women and Theatre: Calling the Shots, Londres, Faber \& Faber, 1984.

46. Helen Crich Chinoy, «Art Versus Business », The Drama Review, vol. 24, n 2, 1980, p. 3-10.

47. Rina Fraticelli, «The invisibility factor: status of women in Canadian theatre », op. cit.

48. Note de la traductrice : Voir ouvrage pré-cité, Dinah Luise Leavitt, Feminist Theatre Groups, Jefferson, N.C, McFarland, 1980.

49. Voir quelques-unes des réinterprétations : Phyllis Rackin, «Androgyny, mimesis, and the marriage of the boy heroine on the English Renaissance stage ", op. cit. ; Jill Dolan, "The Dynamics of desire: sexuality and gender in pornography and performance ", op. cit. ; and Sande Zeig, "The Actor as activator: deconstructing gender through gesture ", Women and Performance, vol. 2, n 2, 1985, p. 12-17.

50. À partir de ses observations de l'onnagata du Kabuki, Peter Hyland suggère que les hommes d'âge mûr, jouaient sans doute « les rôles de femmes d'âge mûr, comme, par exemple, Gertrude, Cléopâtre ou Volumnia ». Pour une explication détaillée, voir "'A Kind of woman': The Elizabethan boy-actor and the Kabuki onnagata ", Theatre research international, vol. 12, n' 1, Printemps 1987, p. 1-8.

51. Lisa Jardine, Still harping on daughters: Women and drama in the age of Shakespeare, Brighton, Sus., Harvester press, Totowa, N.J., Barnes and Noble books, 1983. Cette vision est contestée par Kathleen McLuskie, «The Act, the Role, and the Actor: Boy Actresses on the Elizabethan Stage ", New Theatre Quarterly, vol. 3, n 10, Mai 1987, p. 120-130.

52. Sue-Ellen Case, "Classic drag: the Greek creation of female parts ", Theatre journal, october 1985, p. 321.

53. Note de la traductrice : Charlotte Saunders Cushman est une actrice de théâtre américaine (Boston, 1816-1876), célébrée du temps de son vivant à la fois aux États-Unis et en Angleterre, notamment pour ses interprétations de rôle d'hommes. Lesbienne publiquement assumée, elle forme plusieurs couples avec d'autres femmes artistes. Lydia Webb (1736-1793) interprète anglaise du répertoire comique joue le rôle de Falstaff dans Henry $I V$ de Shakespeare, en 1786 au Haymarket à Londres. Elle est alors considérée obèse.

54. Emma Stebbins, Charlotte Cushman: her Letters and Memories of her Life, Boston, Houghton, Osgood, 1879, p. 60 ; Lyman Horace Weeks, "Some women in doublet 
Questions pour une méthodologie féministe dans l'histoire du théâtre

and hose ", Lippincott's Monthly Magazine, n 57, 1896, p. 88-94 ; et Kathy Flercher, "Planche, vestris, and the tranvestite role: sexuality and gender in Victorian popular theatre ", Nineteenth Century Theatre, n 15, 1, été 1987, p. 9-33.

55. Nancy S. Reinhardt, «New directions for feminist criticism in theatre and the related arts ", op. cit., p. 42-43.

56. Dagmar Höher, «The Composition of music hall audiences, 1850-1900 », dans Music Hall: the Business of Pleasure, ed. Peter Bailey, Milton Keynes, Open University Press, 1986, p. 73-92.

57. Ibid.

58. Lynda Nead, « Feminism, Art History and Cutural Politics », op. cit., p. 124. 